\title{
Spinning gyroscope (bicycle wheel), force, and zero torque exerted by angular momentum
}

\section{Boris Lagutin}

Funding: The author(s) received no specific funding for this work.

Potential competing interests: The author(s) declared that no potential competing interests exist.

\section{Abstract}

This paper (preprint) shows the author's analysis which unveils a problem related to the motion of a spinning gyroscope (bicycle wheel). The classical mechanics formula for torque exerted by the angular momentum of a spinning gyroscope (bicycle wheel) tells that a magnitude of the torque is zero when a gyroscope spins at a uniform (constant) angular speed because a gyroscope angular acceleration is zero. Hence, the torque exerted by a gyroscope angular momentum cannot act against a torque exerted by the Earth gravitation based on the formula. The gravitation pulls a gyroscope (bicycle wheel) down to the Earth surface. As a result, the zero torque, which is exerted by a gyroscope angular momentum, cannot prevent the gyroscope from falling from Position \#1 to Position \#2, see Figure 1. There appears a practical problem - impossibility to calculate a force (torque) needed to change a plane of the spin of the uniformly spinning gyroscope.

Introduction

First of all, let's take a look at a non-spinning bicycle wheel which is suspended by a rope, see Figure 1.

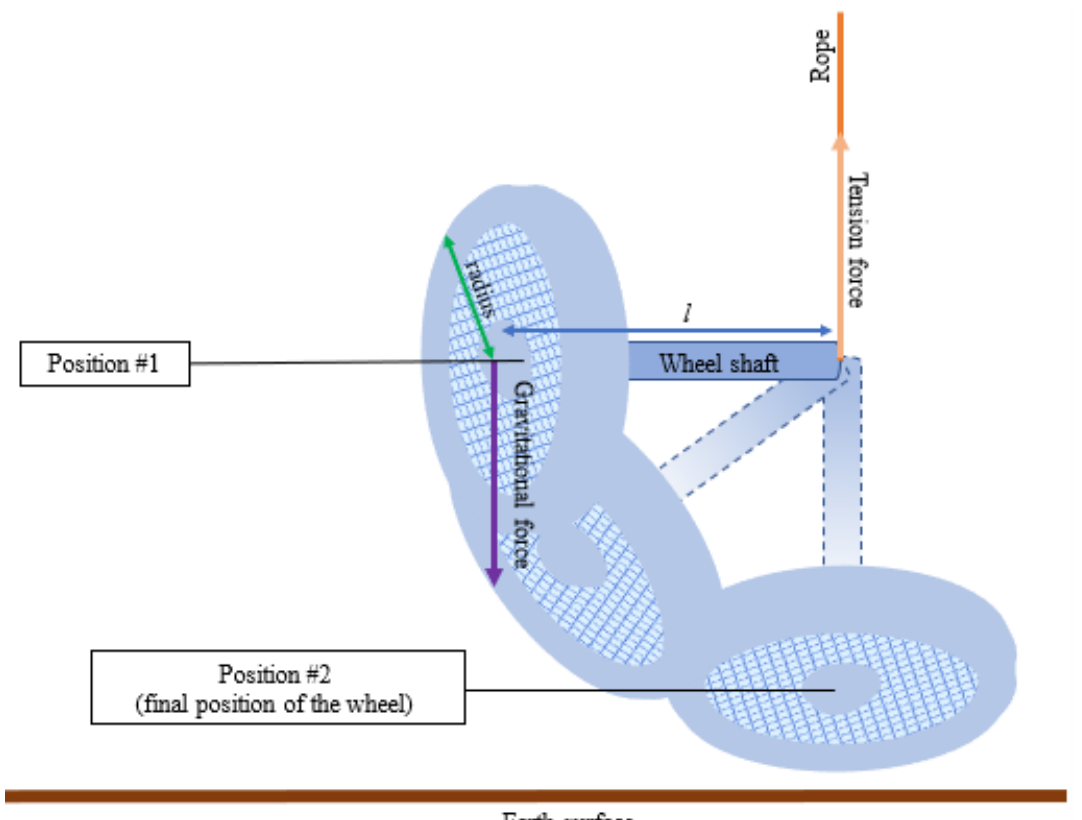

Earth surface 
One end of the wheel shaft is suspended by a rope. If the wheel does not spin at an appropriate angular speed, the wheel falls down from Position \#1 to Position \#2 (see Figure 1) because of the gravitational force. If the wheel spins at an appropriate speed, then the wheel holds its horizontal position and rotates at the horizontal plane (precession), in other words, the wheel shaft stays parallel to the Earth surface as it is shown in Figure 2.

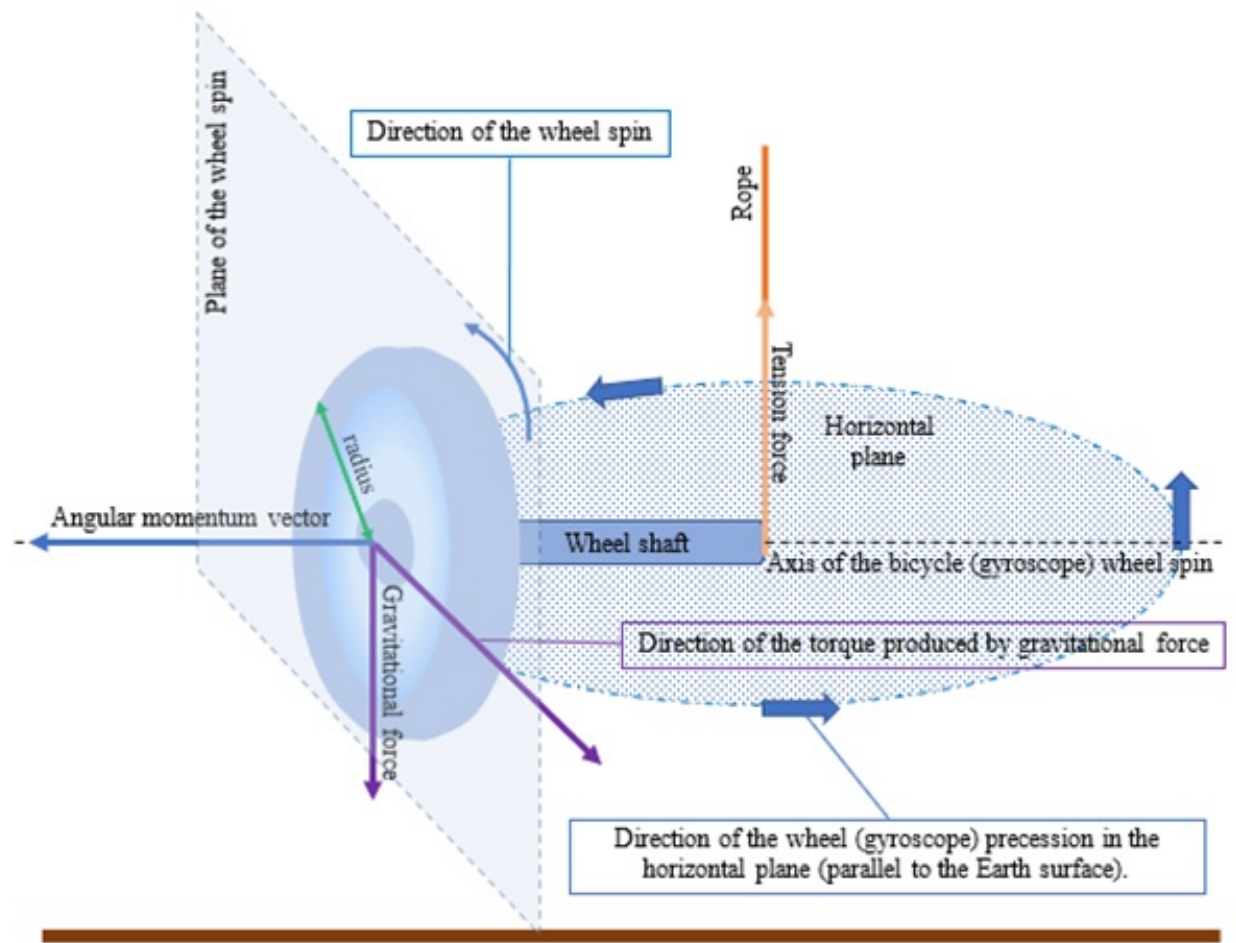

Earth surface

The spinning bicycle wheel works at the same way as that of a gyroscope flywheel. It should be mentioned that a gyroscope flywheel spins, not the whole gyroscope. The same is about the bicycle wheel: a central shaft of the wheel does not spin. The spinning wheel suspended by a rope is demonstrated in this video:

However, the moments of Inertia of the bicycle wheel and of the gyroscope flywheel are different if the flywheel is disk [1]:

$$
I_{\text {bicycle wheel }}=m_{\text {bicycle wheel }} R_{\text {bicycle wheel }}^{2},
$$

and

$$
I_{\text {gyroscope flywheel }}=\left(m_{\text {gyroscope flywheel }} R_{\text {gyroscope flywheel }}^{2}\right) / 2, \text { (2) }
$$

where $m$ symbolizes a mass of the bicycle wheel or of the gyroscope flywheel, $R$ is a radius of the bicycle wheel or of the gyroscope flywheel.

Next, analyze which forces and torques act when the non-spinning bicycle wheel (gyroscope) is suspended by a rope above the Earth surface, see Figure 3. 


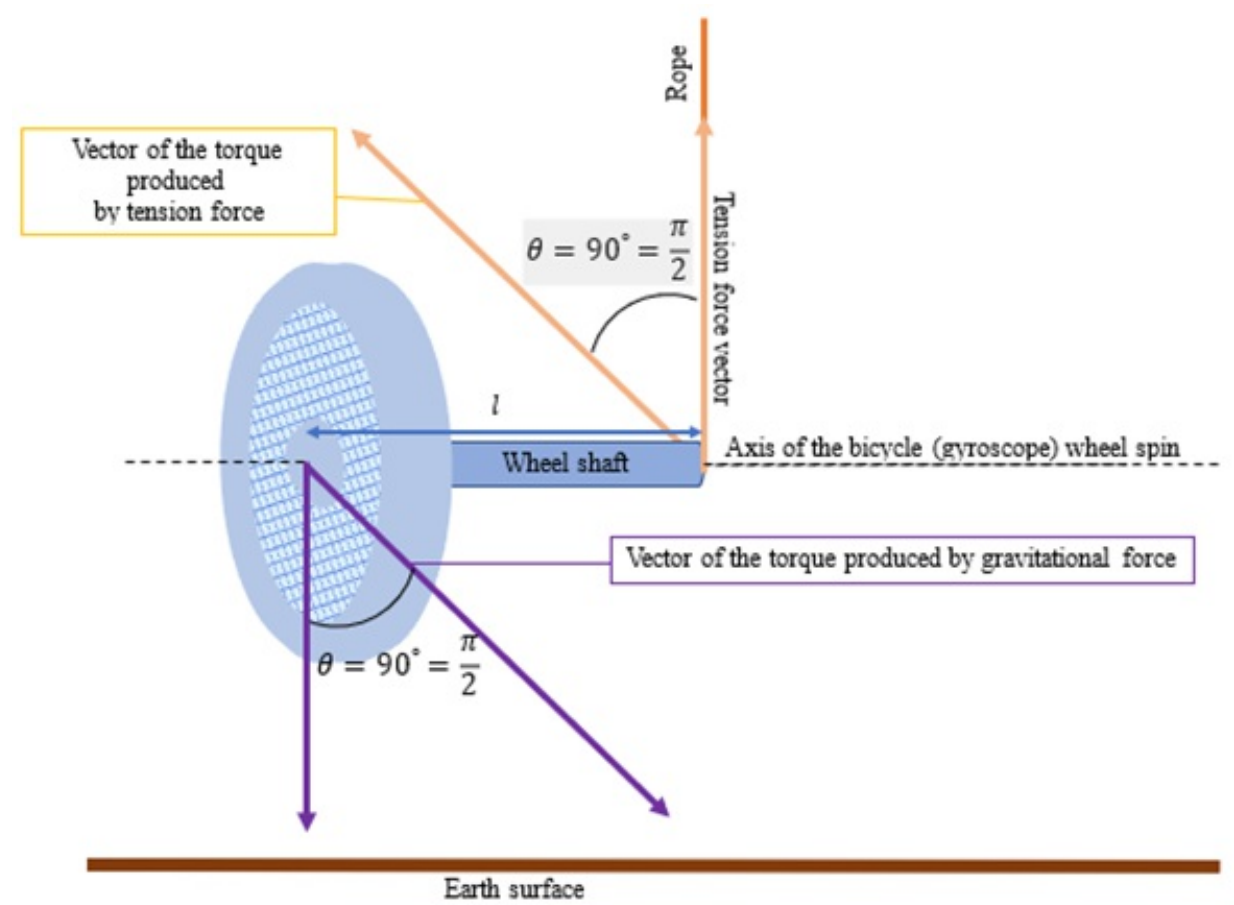

One can see that there are a tension force and a gravitational force which act at the opposite directions to each other. The tension force pulls the bicycle wheel up, but the gravitational force pulls it down. Notice both the gravitational force and the tension force exert torques when the wheel does not spin and the wheel shaft is located parallel to the Earth surface, see Figure 3. Hence, the bicycle wheel falls down, even though the magnitude of the gravitational force torque should be equal to the magnitude of the tension force torque in Position \#1 in Figure 1:

$$
\tau_{\text {torgue exerted by tension force }}=\tau_{\text {torque exerted by gravitational force }}=m g l \text {, }
$$

where $m$ is a mass of the bicycle wheel or of the gyroscope, $g$ is the gravitational acceleration 9.8 meters per second squared , I is a length of the wheel shaft. Notice that the wheel falls down if it is released at Position \#1. It is time to analyze vectors of which forces and torques act when the bicycle wheel spins and its shaft is located parallel to the Earth surface.

There are the gravitational and tension forces. Also, there are the torques exerted by the gravitational and tension forces which are vectors, see Figure 4. 


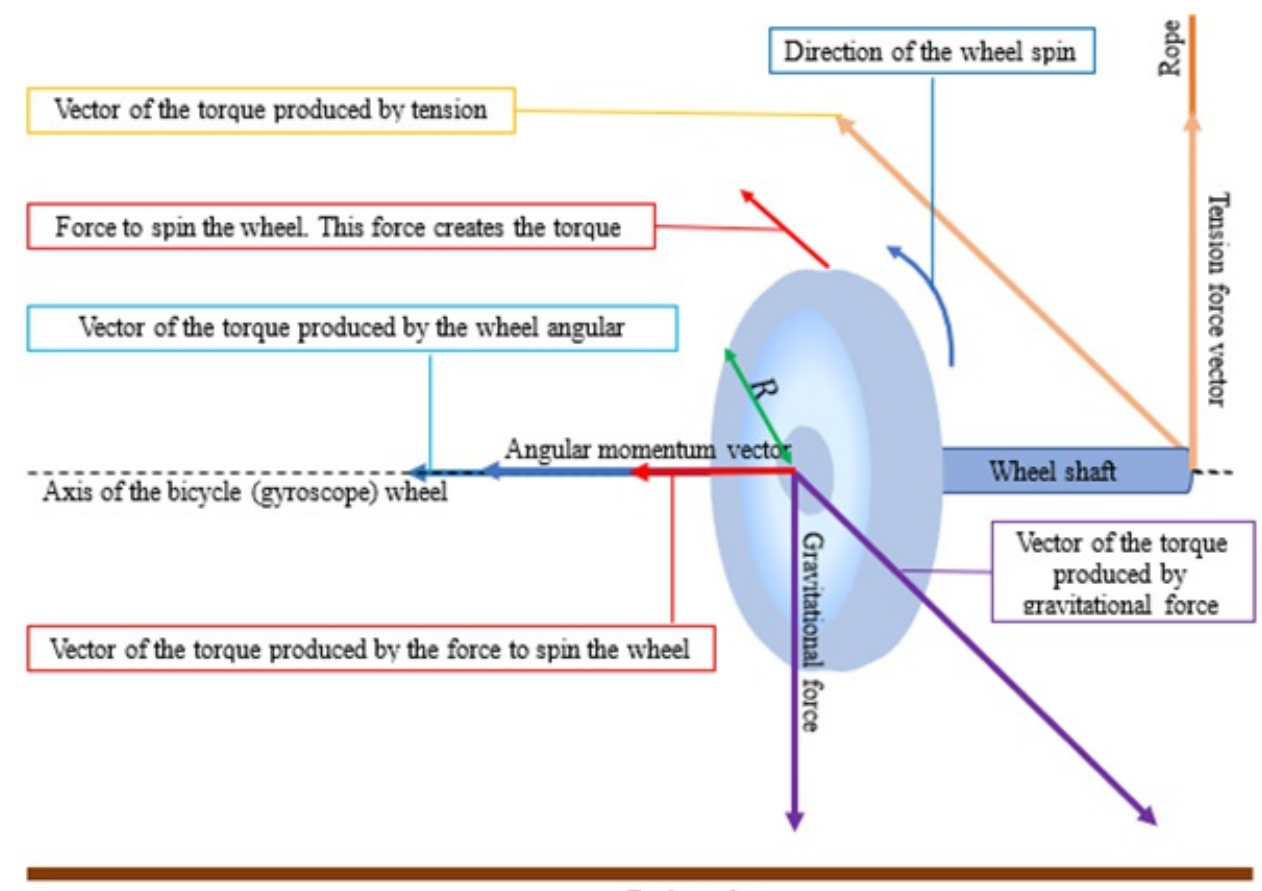

Earth surface

Also, there is a torque exerted by a force to spin the bicycle wheel:

$$
\tau_{\text {exerted by force to spin wheel }}=R_{\text {wheel }} F_{\text {force to spin wheel }} \text {, }
$$

where $R$ is a radius of the bicycle wheel or of the gyroscope flywheel. This torque vector points at the same direction as that of the wheel spin angular momentum vector as it is shown in Figure 4. The magnitude of the torque exerted by the wheel spin angular momentum is defined as the derivative of the wheel spin angular momentum with respect to time [2]:

$$
\begin{gathered}
\tau_{\text {(exerted by spin angularmomentum })}=d L / d t=d\left(I_{\text {whee }} \omega_{\text {wheelspin }}\right) / d t= \\
=d\left(m_{\text {wheel }} R_{\text {wheel }}^{2} \omega_{\text {wheel spin }}\right) / d t= \\
=\left(d\left(m_{\text {wheel }} R_{\text {wheel }}^{2}\right) / d t\right) \omega_{\text {wheelspin }}+m_{\text {wheel }} R_{\text {wheel }}^{2} \alpha_{\text {wheelspin }} \text {, (5) }
\end{gathered}
$$

where $L$ is the bicycle wheel spin angular momentum, $m$ is the bicycle wheel mass, $R$ is its radius, $w$ is its angular (spin) speed, $a$ is its angular (spin) acceleration. Now, answer the question: "What prevents the wheel from falling down from Position \#1 to Position \#2 when the wheel spins?"

The spinning bicycle wheel or the spinning gyroscope suspended by a rope does not fall down because they have the spin angular momentum. But, how the spin angular momentum may prevent the wheel (gyroscope) suspended by a rope from falling down? The answer is that the wheel spin angular momentum exerts a torque which acts against the torque exerted by the gravitational force. Therefore, the torque exerted by the wheel spin angular momentum must be equal to or larger than the torque exerted by the gravitational force in order to prevent the wheel from falling down from Position \#1 to Position \#2, see Figures 1, 3, 4:

$$
\begin{aligned}
& \tau_{\text {exerted by wheel spin angularmomentum }} \geq \tau_{\text {exerted by gravitational force }} \text {, } \\
& \left(\left(d\left(m_{\text {wheel }} R_{\text {wheel }}^{2}\right) / d t\right) \omega_{\text {wheel spin }}+m_{\text {wheel }} R_{\text {wheel }}^{2} \alpha_{\text {wheel spin }}\right) \geq m g l
\end{aligned}
$$


Now, take a careful look at the formula (5) for the torque exerted by the wheel spin angular momentum. The bicycle wheel mass $m$ is constant. The bicycle wheel radius $R$ is constant. The bicycle wheel spin angular speed $w$ may change if there is the spin angular acceleration $a$. And, consider a situation when the bicycle wheel has an appropriate constant (uniform) angular speed. If the wheel has a constant angular speed, then all the terms, including the angular acceleration, of the formula (5) for the torque become zeros. As known, the derivatives of constants are always equal to zero:

$$
\begin{gathered}
\tau_{\text {exerted by angularmomentum }}=d L / d t=d\left(I_{\text {wheel }} \omega_{\text {wheel spin }}\right) / d t=d\left(m_{\text {wheel }} R_{\text {wheel }}^{2} \omega_{\text {wheel spin }}\right) / d t= \\
=\left(d\left(m_{\text {wheel }} R_{\text {wheel }}^{2}\right) / d t\right) \omega_{\text {wheel spin }}+m_{\text {wheel }} R_{\text {wheel }}^{2} \alpha_{\text {wheel spin }}=0+0=0, \text { (8) }
\end{gathered}
$$

The similar formula is applied to calculate the torque exerted by the gyroscope flywheel if the flywheel is a disk. However, the disk moment of Inertia is defined by the formula

$$
I_{\text {flywheel }}=\left(m_{\text {flywheel }} R_{\text {flywheel }}^{2}\right) / 2 \text {, }
$$

therefore, if the flywheel angular acceleration is zero:

$$
\tau_{\text {exerted by flywheel angularmomentum }}=d\left(I_{\text {flywheel }} w_{\text {flywheel }} \text { spin }\right) / d t=0+0=0 .
$$

As a result, the torque exerted by the wheel angular momentum is equal to zero when the wheel spins at the constant angular speed! Therefore, the torque, which is exerted by the angular momentum, cannot prevent the bicycle wheel (gyroscope) from falling down when the wheel (flywheel) spins at the constant angular speed based on the equations (8) and (10). However, the bicycle wheel or the gyroscope does not fall down from Position \#1 to Position \#2, even if the wheel or the gyroscope flywheel spins at the appropriate constant angular speed. The last statement is also supported by the formulas for the bicycle wheel or gyroscope precession angular speeds [3]:

$$
\begin{gathered}
\omega_{\text {precession of bicycle wheel }}=\tau_{\text {exerted by gravitational force }} / L_{\text {wheel spin angularmomentum }}=\left(I m_{\text {wheel }} g\right) /\left(I_{\text {wheel }} \omega_{\text {wheelspin }}\right),(1 \\
\omega_{\text {precession of gyroscope }}=\tau \text { exerted by gravitational force } / L_{\text {flywheel spin angularmomentum }}=\left(I m_{\text {flywheel }} g\right) /\left(I_{\text {flywhee }} / \omega_{\text {flywheels spin }}\right) .
\end{gathered}
$$

As it is seen from the formula for the bicycle wheel precession speed (11), this formula defines the wheel precession angular speed by using the wheel spin angular speed only. The formula for the gyroscope precession speed (12) defines the gyroscope precession angular speed by using the flywheel spin angular speed only. In other words, no any angular acceleration is needed. Based on these formulas, the precession exists at the constant angular speeds. Hence, if the precession happens, the wheel (suspended by a rope) must hold its horizontal position with respect to the Earth surface at appropriate angular speeds. In other words, the bicycle wheel or the gyroscope should not fall down immediately. It should be mentioned that the uniformly spinning gyroscope (bicycle wheel) does not fall down from Position \#1 to Position \#2 under the gravitational force pull, even if one mechanically stops the gyroscope (bicycle wheel) precession; in other words, even if changing a direction of the spin angular momentum vector by precession is stopped. For example, one could stop the gyroscope (bicycle wheel) precession with one's finger.

Finally, consider the uniformly spinning gyroscope in the microgravity, in particular inside the International Space Station. A uniformly spinning gyroscope does not have any precession onboard the International Space Station. In other words, the direction of the vector of the gyroscope flywheel spin angular momentum does not change. The uniformly 
spinning gyroscope stays at the same position without any precession in this video

The astronaut may easily change the direction of the non-spinning gyroscope shaft. However, the astronaut cannot change the direction (spin axis direction) of the uniformly spinning gyroscope shaft because the gyroscope simply flies away from the astronaut's finger keeping the same plane of the flywheel spin, see the video and Figure 5.

\section{Disposal \#1}

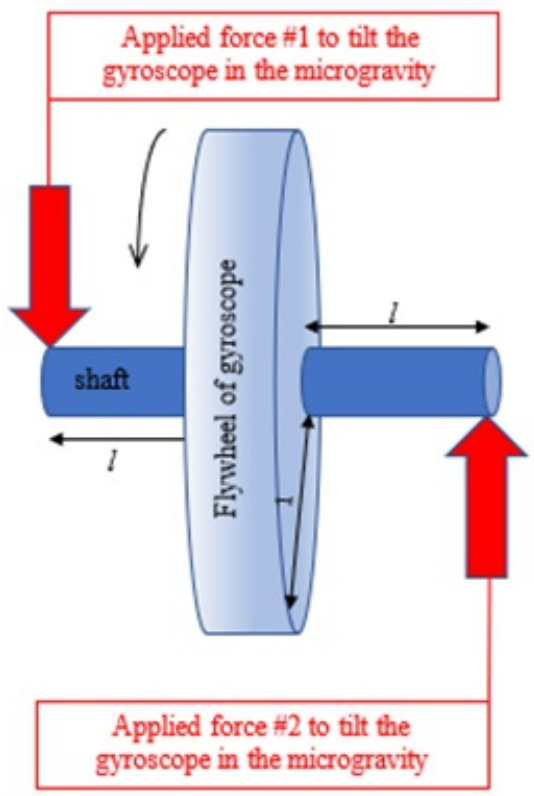

\section{Disposal $\# 2$}

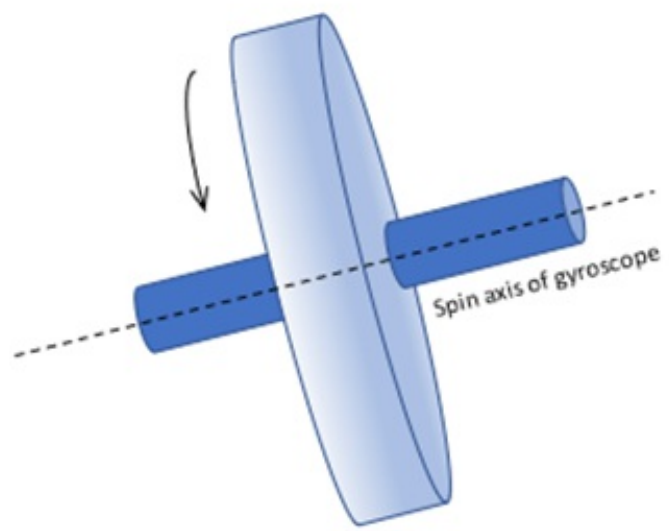

In other words, the astronaut should apply some significant force to change the gyroscope flywheel spin plane. That is, a significant torque must be exerted on the gyroscope shaft to change the gyroscope flywheel spin plane. It is possible to execute only if the astronaut exerts an equal force on each end of the gyroscope shaft at opposite directions as shown in Figure 5, otherwise, the gyroscope just flies away keeping the same plane of its spin.

The only torque, which acts against the torque applied by the astronaut, is the torque exerted by the gyroscope flywheel spin angular momentum. But, this torque is equal to zero based on the formula (5) if the gyroscope flywheel spins at any uniform angular speed (no any angular acceleration). Moreover, the vector of the flywheel spin angular momentum does not change its direction because there is no any precession. One can see that the spinning gyroscope holds its initial plane of its flywheel spin when the astronaut pushes the gyroscope shaft, watch the video.

\section{Conclusion}

There is a practical problem how to calculate a force necessary to change the spin plane of the uniformly spinning gyroscope flywheel (bicycle wheel) via the spin angular momentum. It also appears to be impossible to calculate the torque exerted by the spin angular momentum if the gyroscope flywheel (bicycle wheel) spins at the uniform (constant) angular speed in the International Space Station. Therefore, the classical mechanics formulas do not describe the situation when the spin angular speed is constant.

It should be mentioned that the torque, which is exerted by the gravitational force on the gyroscope (bicycle wheel), 
depends on a length of the wheel shaft I, but the torque, which is exerted by the gyroscope (bicycle wheel) spin angular momentum, does not depend on a length of the wheel shaft I, see Figure 1.

References:

1. Dr. Paul Peter Urone, Dr. Roger Hinrichs, Dr. Kim Dirks, Dr. Manjula Sharma, College Physics (Rice University, OpenStax, 2017), Chapter 10, pp. 347-389, https://openstax.org/details/books/College-Physics 2. Dr. Paul Peter Urone, Dr. Roger Hinrichs, Dr. Kim Dirks, Dr. Manjula Sharma, College Physics (Rice University, OpenStax, 2017), Chapter 10, pp. 347-389, https://openstax.org/details/books/College-Physics 3. Robert G. Brown, Introductory Physics I, Elementary Mechanics (Duke University Physics Department, Durham, 1993, 2007, 2013), pp. 352-364, https://webhome.phy.duke.edu/ rgb/Class/intro physics 1/intro physics 1.pdf 